See Article page 970.

\section{Commentary: To fix or not to fix? That is the question for mitral and aortic valve surgery during left ventricular assist devices implantation}

\author{
Edward G. Soltesz, MD, MPH
}

The decision to intervene on valvular lesions during durable left ventricular assist device (LVAD) implantation remains an area of considerable debate. Some argue that most valvular lesions short of severe aortic valve regurgitation or mitral stenosis do not require correction at the time of LVAD insertion. The lack of randomized trial data in this area along with the additional risk engendered by cardioplegic arrest and longer cardiopulmonary bypass time lend support to this more conservative surgical strategy. On the other hand, evolving evidence is suggesting that certain groups of patients might benefit from more aggressive valvular interventions at the time of LVAD surgery. Smood and colleagues ${ }^{1}$ present a cogent review of their institutional bias and practice on when to intervene on aortic and mitral valve disease at the time of LVAD surgery.

Although surgical correction of moderate or greater degrees of aortic insufficiency (AI) at the time of LVAD is generally an accepted practice, there remains ongoing controversy about management of mild $\mathrm{AI}^{2}$ Acknowledging that mild preimplant AI has a much higher chance of worsening after LVAD implant, the authors rightly advocate for either aortic valve replacement (AVR) or aortic valve closure (eg, Park stitch) for mild AI. ${ }^{3}$ AVR allows for

\footnotetext{
From the Department of Thoracic and Cardiovascular Surgery and Kaufman Center for Heart Failure and Recovery, Heart and Vascular Institute, Cleveland Clinic, Cleveland, Ohio.

Disclosures: Dr Soltesz has been a consultant for Abbott, Abiomed, and Atricure.

The Journal policy requires editors and reviewers to disclose conflicts of interest and to decline handling or reviewing manuscripts for which they may have a conflict of interest. The editors and reviewers of this article have no conflicts of interest.

Received for publication Feb 10, 2021; revisions received Feb 10, 2021; accepted for publication Feb 11, 2021; available ahead of print Feb 18, 2021.

Address for reprints: Edward G. Soltesz, MD, MPH, Department of Thoracic and Cardiovascular Surgery, Cleveland Clinic, 9500 Euclid Ave, Desk J4-1, Cleveland, OH (E-mail: soltese@ccf.org).

J Thorac Cardiovasc Surg 2022;164:979-80

$0022-5223 / \$ 36.00$

Copyright (c) 2021 by The American Association for Thoracic Surgery

https://doi.org/10.1016/j.jtcvs.2021.02.031
}

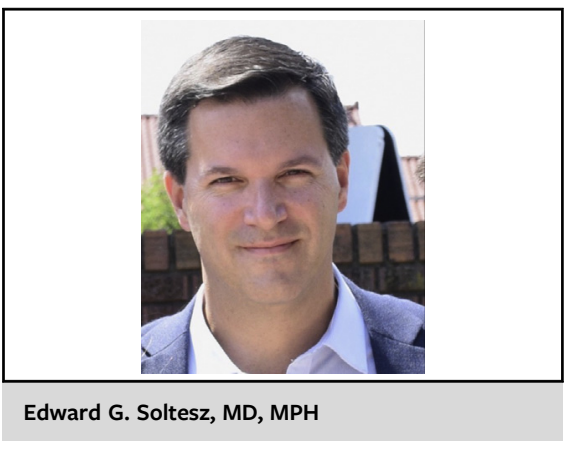

CENTRAL MESSAGE

Evolving evidence suggests that concomitant aortic and mitral valve surgery at the time of LVAD implant is reasonable in carefully selected patients.

valve-in-valve transcatheter AVR if bioprosthetic AI develops, whereas aortic valve closure obviates any future transvalvular intervention. Surgeons must remember that meticulous myocardial protection during aortic valve procedures is crucial to prevent postimplant right ventricular dysfunction.

The optimal treatment of severe mitral regurgitation (MR) at the time of LVAD implant, on the other hand, remains a matter of significant debate. Although MR typically improves after LVAD implant in a majority of patients, a small but important subset continue to have MR. Studies examining the clinical significance of this residual MR and the overall effect of mitral repair have come to disparate conclusions and provide no data-driven consensus on best practices. ${ }^{4}$ As the authors correctly note, patients with significant right ventricular dysfunction and/or pulmonary hypertension might benefit from mitral repair because residual MR in these patient populations portend poor outcomes. Smood and colleagues ${ }^{1}$ do not programmatically recommend mitral intervention at the time of LVAD implant unless there is a reasonable expectation of recovery and LVAD explant. This may have been a reasonable approach in the past, but today's bridge-to-transplant and destinationtherapy LVAD patients are living much longer with their devices due to the new United Network for Organ Sharing adult heart allocation prioritizations and the improved outcomes with magnetically levitated centrifugal pumps. In light of this, it may now be reasonable to address severe MR at the time of LVAD implant to reduce pulmonary 
vascular resistance as well as the risk of long-term right heart failure in select patients.

Current guidelines surrounding indications for concomitant valvular surgery during LVAD implantation clearly suffer from a lack of high-quality evidence. Until a randomized controlled trial or large clinical registry can provide evidence-based practice recommendations, we are left with expert opinions provided by thought-leaders in the field. The authors make a cogent argument to be aggressive with AI, but they don't have the same enthusiasm for aggressive treatment of MR. Until more data emerge from this patient population, the question remains: To fix or not to fix?

\section{References}

1. Smood B, Han JJ, Helmers M, Atluri P. Invited expert opinion: mitral and aortic valve surgery during left ventricular assist device implantation. J Thorac Cardiovasc Surg. 2022;164:970-7.

2. Kirklin JK, Pagani FD, Goldstein DJ, John R, Rojers JG, Atluri P, et al. American Association for Thoracic Surgery/International Society for Heart and Lung Transplantation guidelines on selected topics in mechanical circulatory support. J Heart Lung Transplant. 2020;39:187-219.

3. Tanaka Y, Nakajima T, Fischer I, Wan F, Kotkar K, Moon MR, et al. The impact of uncorrected mild aortic insufficiency at the time of left ventricular assist device implantation. J Thorac Cardiovasc Surg. 2020;160: 1490-500.e3.

4. Tang PC, Haft JW, Romano MA, Bitar A, Hasan R, Palardy M, et al. Right ventricular function and residual mitral regurgitation after left ventricular assist device implantation determines the incidence of right heart failure. J Thorac Cardiovasc Surg. 2020;159:897-905.e4. 